Supporting Information

For

\title{
Intersystem Crossing Mediated by Photoinduced Intramolecular Charge Transfer: Julolidine-Anthracene Molecules with Perpendicular $\pi$ Systems
}

Department of Chemistry, Argonne-Northwestern Solar Energy Research (ANSER) Center, and International Institute of Nanotechnology, Northwestern University, Evanston, Illinois 60208-3113

Zachary E. X. Dance, Sarah M. Mickley, Thea M. Wilson, Annie Butler Ricks, Amy M. Vega, Mark A. Ratner*, and Michael R. Wasielewski*

e-mail: $\underline{\text { m-wasielewski@northwestern.edu }}$

\section{Synthesis}

\section{General}

Proton nuclear magnetic resonance spectra were recorded on a Mercury 400 or Inova 500 NMR spectrometer using TMS as an internal standard. Laser desorption mass spectra were obtained with a Micromass 70-SE-4F or Micromass 70-VSE high resolution mass spectrometer. Solvents and reagents were used as received except for MTHF, which was purified by distilling over $4 \AA$ molecular sieves, passing through a column of basic alumina and filtering through a $0.2 \mu \mathrm{m}$ PTFE filter. Flash and thin-layer chromatography was performed using Sorbent Technologies (Atlanta, GA) silica gel. All solvents were spectrophotometric grade. Toluene was purified by passing it through a series of $\mathrm{CuO}$ and alumina columns (GlassContour). Chloroform was washed three times with deionized water, dried over sodium sulfate, and then passed through a column of basic alumina. 


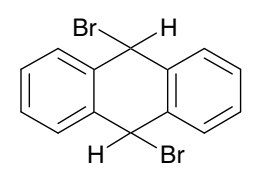

9,10-dibromo-9,10-dihydroanthracene. ${ }^{1}$ Anthracene $(5.012 \mathrm{~g}, 28.12 \mathrm{mmol})$ was placed in a flame-dried $250 \mathrm{ml}$ round bottom flask with $55 \mathrm{ml}$ dry carbon disulfide. The reaction flask was cooled to $0^{\circ} \mathrm{C}$ with a water / ice bath under a nitrogen atmosphere. Bromine $(1.3 \mathrm{ml}, 25.30$ mmol, in $20 \mathrm{ml}$ carbon disulfide) was added dropwise over $48 \mathrm{~min}$. After stirring for $30 \mathrm{~min}$. at $0^{\circ} \mathrm{C}$ the reaction contents were filtered through a glass-fritted(fine) büchner funnel, washed with cold carbon disulfide then cold diethyl ether. The tan powder was dried for 10 min by vacuum suction and used as-is, $7.968 \mathrm{~g}(23.57 \mathrm{mmol}), 83.8 \%$.

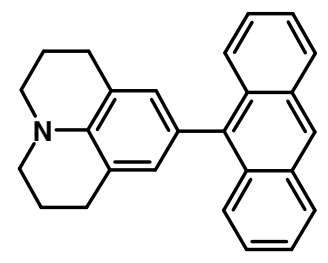

4-(9-anthracenyl)-julolidine (J-An). 9,10-Dibromo-9,10-dihydroanthracene ${ }^{1}$ (1.199 g, 3.57 mmol) was placed in a flame-dried $100 \mathrm{ml}$ round bottom flask with $20 \mathrm{ml}$ dry chloroform (ethanol free). The reaction flask was cooled to $0^{\circ} \mathrm{C}$ using a water / ice bath under a nitrogen atmosphere. Julolidine (1.232 $\mathrm{g}, 7.12 \mathrm{mmol}$, in $5 \mathrm{ml}$ dry chloroform) was added dropwise over 35 min. to the cooled reaction mixture. After the addition was complete the reaction was stirred at $0^{\circ} \mathrm{C}$ for an additional $2.5 \mathrm{hrs}$. then warmed to room temperature overnight with continuous stirring under nitrogen. The solvent was removed on a rotary evaporator and the residue purified via flash column chromatography on silica gel. Elution with $25 \%$ hexanes in methylene chloride (v/v) yielded (J-An) as a yellowish powder, $0.282 \mathrm{~g}(0.808 \mathrm{mmol}), 22.6 \% . \mathrm{C}_{26} \mathrm{H}_{23} \mathrm{~N} .{ }^{1} \mathrm{H}$ NMR 
$\left(\mathrm{CDCl}_{3}\right) \delta: 8.419(\mathrm{~s}, 1 \mathrm{H}), 8.013(\mathrm{~d}, \mathrm{~J}=8 \mathrm{~Hz}, 2 \mathrm{H}), 7.899(\mathrm{~d}, \mathrm{~J}=8.4 \mathrm{~Hz}, 2 \mathrm{H}), 7.436(\mathrm{t}, \mathrm{J}=7.2 \mathrm{~Hz}$, 2H), 7.348 (m, 2H), 6.849 (s, 2H), 3.244 (bs, 4H), 2.828 (bs, 4H), 2.080 (bs, 4H).

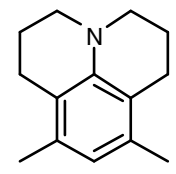

3,5-dimethyljulolidine. 3,5-Dimethylaniline (4.019 g, $33.17 \mathrm{mmol}$ ), 1-bromo-3-chloropropane (113.567 g, $721.34 \mathrm{mmol})$, sodium carbonate $\left(14.576 \mathrm{~g}, 137.95 \mathrm{mmol}\right.$, oven dried at $300^{\circ} \mathrm{C}$ for 4 hours) and $4 \AA$ molecular sieves $\left(3.5 \mathrm{~g}\right.$ activated at $\left.300^{\circ} \mathrm{C}\right)$ were added to a flame-dried $250 \mathrm{ml}$ round bottom flask under a nitrogen atmosphere. The following heating rate was applied: $1 \mathrm{hr}$. at $80^{\circ} \mathrm{C}, 2 \mathrm{hr}$. at $100^{\circ} \mathrm{C}, 12 \mathrm{hr}$. at $160^{\circ} \mathrm{C}$. The reaction mixture was cooled to room temperature, diluted with $200 \mathrm{ml}$ methylene chloride, then washed successively with: $500 \mathrm{ml}$ of $10 \% \mathrm{HCl}$, twice with $500 \mathrm{ml}$ water, once with $500 \mathrm{ml}$ of $10 \% \mathrm{NaOH}$ and twice with $500 \mathrm{ml}$ water. The organic fraction was dried over sodium sulfate and then flash column chromatographed on silica gel using a solvent gradient beginning with $40 \%$ hexanes in methylene chloride (v/v) and ending with $100 \%$ methylene chloride providing 3,5-dimethyljulolidine as a white crystalline powder, $5.669 \mathrm{~g}$ (28.16 mmol), 84.9\%. $\mathrm{C}_{14} \mathrm{H}_{19} \mathrm{~N}$ HRMS-ESI (m/z): [M+e] 201.1517 (calc. 201.1517). ${ }^{1} \mathrm{H} \mathrm{NMR}\left(\mathrm{CDCl}_{3}\right) \delta: 6.380(\mathrm{~s}, 1 \mathrm{H}), 3.065(\mathrm{t}, \mathrm{J}=5.5 \mathrm{~Hz}, 4 \mathrm{H}), 2.630(\mathrm{t}, \mathrm{J}=6.8 \mathrm{~Hz}, 4 \mathrm{H}), 2.120(\mathrm{~s}$, 6H), $2.020(\mathrm{~m}, 4 \mathrm{H})$.

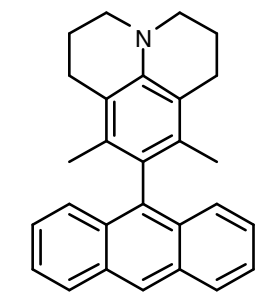

3,5-dimethyl-4-(9-anthracenyl)julolidine (DMJ-An). 9,10-Dibromo-9,10-dihydroanthracene $(1.423 \mathrm{~g}, 4.21 \mathrm{mmol})$ was placed in a flame-dried $100 \mathrm{ml}$ round bottom flask with $20 \mathrm{ml}$ dry 
chloroform (ethanol free). The reaction flask was cooled to $0^{\circ} \mathrm{C}$ using a water / ice bath under a nitrogen atmosphere. 3,5-Dimethyljulolidine (1.643 g, $8.17 \mathrm{mmol}$, in $10 \mathrm{ml}$ dry chloroform) was added dropwise over $35 \mathrm{~min}$. to the cooled reaction mixture. After the addition was complete the reaction was stirred at $0^{\circ} \mathrm{C}$ for an additional 2.5 hours and then warmed to room temperature overnight with continuous stirring under nitrogen. The solvent was removed on a rotary evaporator and the residue purified via flash column chromatography on silica gel. Elution with 40\% hexanes in methylene chloride (v/v) yielded DMJ-An as a yellowish powder, $0.301 \mathrm{~g}$ (0.080 mmol), 18.9\%. $\mathrm{C}_{28} \mathrm{H}_{27} \mathrm{~N}$ HRMS-ESI (m/z): $[\mathrm{M}+\mathrm{H}]^{+} 378.2219$ (calc. 378.2222$) .{ }^{1} \mathrm{H}$ NMR $\left(\mathrm{CDCl}_{3}\right) \delta: 8.448(\mathrm{~s}, 1 \mathrm{H}), 8.044(\mathrm{~d}, \mathrm{~J}=8.5 \mathrm{~Hz}, 2 \mathrm{H}), 7.593(\mathrm{~d}, \mathrm{~J}=8.7 \mathrm{~Hz}, 2 \mathrm{H}), 7.437(\mathrm{t}, \mathrm{J}=8.5$ $\mathrm{Hz}, 2 \mathrm{H}), 7.315(\mathrm{~m}, 2 \mathrm{H}), 3.180(\mathrm{t}, \mathrm{J}=5.5 \mathrm{~Hz}, 4 \mathrm{H}), 2.732(\mathrm{t}, \mathrm{J}=6.8 \mathrm{~Hz}, 4 \mathrm{H}), 2.120(\mathrm{~m}, 4 \mathrm{H}), 1.510$ $(\mathrm{s}, 6 \mathrm{H})$.

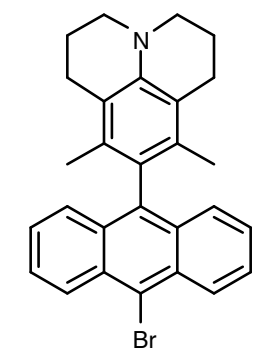

3,5-dimethyl-4-(10-bromoanthracenyl)julolidine. DMJ-An (0.139 g, 0.369 mmol) was added to a $50 \mathrm{ml}$ round bottom flask with $20 \mathrm{ml}$ acetic acid. Sonication and slight heating was necessary to fully dissolve the starting material. Using a disposable Pasteur pipette, seven drops of bromine were added slowly. There was an immediate color change to dark brown and a slight precipitate. Continued stirring for an additional 15 minutes yielded a light tan solution. The reaction mixture was diluted with methylene chloride and washed twice with water and then once with a $\mathrm{NaHCO}_{3}$ solution. Purification via a plug of silica gel using methylene chloride (v/v) provided 3,5-dimethyl-4-(10-bromoanthracenyl)julolidine as a yellowish solid, 0.100 $\mathrm{g}$ (0.219 
mmol), $60.0 \% . \mathrm{C}_{28} \mathrm{H}_{26} \mathrm{BrN}$ HRMS-ESI (m/z): $[\mathrm{M}+\mathrm{H}]^{+} 456.1305$ (calc. 456.1327). ${ }^{1} \mathrm{H}$ NMR $\left(\mathrm{CDCl}_{3}\right) \delta: 8.585(\mathrm{~d}, \mathrm{~J}=8.8 \mathrm{~Hz}, 2 \mathrm{H}), 7.693-7.535(\mathrm{~m}, 4 \mathrm{H}), 7.343(\mathrm{dd}, \mathrm{J}=8.7 \mathrm{~Hz}, 2 \mathrm{H}), 3.181$ (t, J = 5.5 Hz, 4H), $2.712(\mathrm{t}, \mathrm{J}=6.8 \mathrm{~Hz}, 4 \mathrm{H}), 2.113(\mathrm{~m}, 4 \mathrm{H}), 1.486(\mathrm{~s}, 6 \mathrm{H})$.

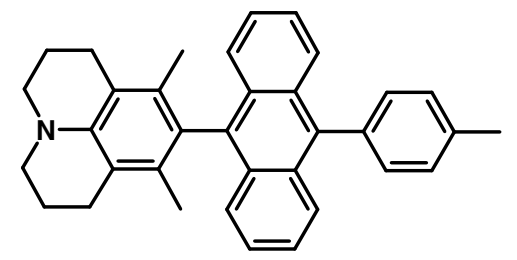

3,5-dimethyl-4-(10-tolyl-anthracenyl)-julolidine (DMJ-An-tol). 3,5-dimethyl-4-(10-bromoanthracenyl)-julolidine $(0.142 \mathrm{~g}, 0.312 \mathrm{mmol})$ was placed in a flame-dried $50 \mathrm{ml}$ round bottom flask with $20 \mathrm{ml}$ toluene. Tributyl-p-tolyl-stannane ${ }^{2}(0.36 \mathrm{~g}, 0.934 \mathrm{mmol})$ and palladium (0) tetrakistriphenylphosphine $(0.025 \mathrm{~g}, 0.02 \mathrm{mmol})$ were added to the solution. The reaction flask was heated to $80^{\circ} \mathrm{C}$ for sixteen hours under a nitrogen atmosphere, without the presence of light. The solvent was removed on a rotary evaporator and the residue purified via column chromatography on silica gel. Elution with $20 \%$ hexanes in methylene chloride yielded DMJAn-tol with a few impurities. Preparative TLC on silica gel using $40 \%$ hexanes in methylene chloride as the eluent was required for complete purification. $\mathrm{C}_{35} \mathrm{H}_{33} \mathrm{~N}$ HRMS-ESI $(\mathrm{m} / \mathrm{z})$ : $[\mathrm{M}+\mathrm{H}]^{+} 468.2695$ (calc. 468.2691). ${ }^{1} \mathrm{H} \mathrm{NMR}\left(\mathrm{CDCl}_{3}\right) \delta: 7.728(\mathrm{~d}, \mathrm{~J}=8.9 \mathrm{~Hz}, 2 \mathrm{H}), 7.399(\mathrm{~m}$, 6H), 7.300 (m, 4 H), 3.193 (br s, 4H), 2.754 (br s, 4H), 2.541 (s, 3H), 2.142 (br s, 4H), 1.522 (s, $6 \mathrm{H})$. 


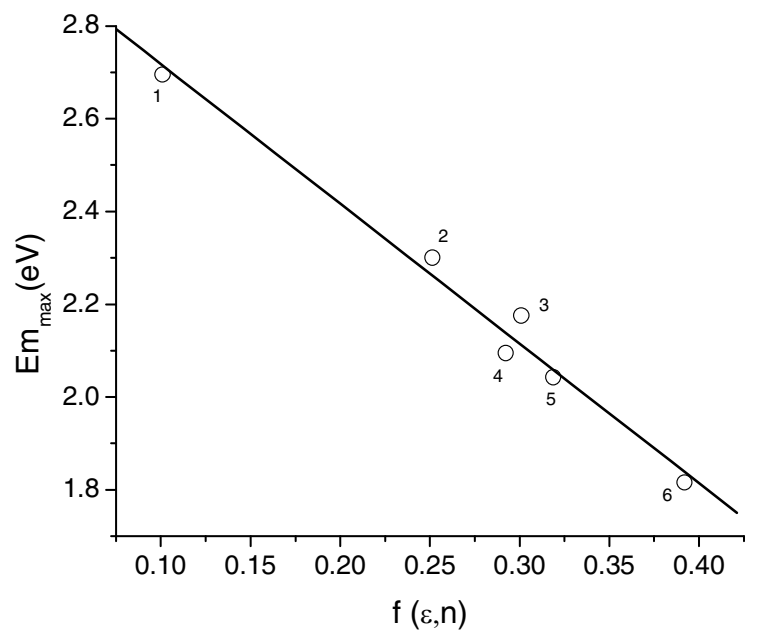

Figure S1. Lippert-Mataga for DMJ-An $\left(\mathrm{R}^{2}=0.981\right.$ where $\mathrm{f}(\varepsilon, \mathrm{n})=\left[(\varepsilon-1) /(2 \varepsilon+1)-0.5 \mathrm{x}\left(\mathrm{n}^{2}-\right.\right.$ $\left.1) /\left(2 n^{2}+1\right)\right]$. The solvents are (1) methylcyclohexane, (2) diethyl ether, (3) 2methyltetrahydrofuran, (4) ethyl acetate, (5) methylene chloride, and (6) acetonitrile.

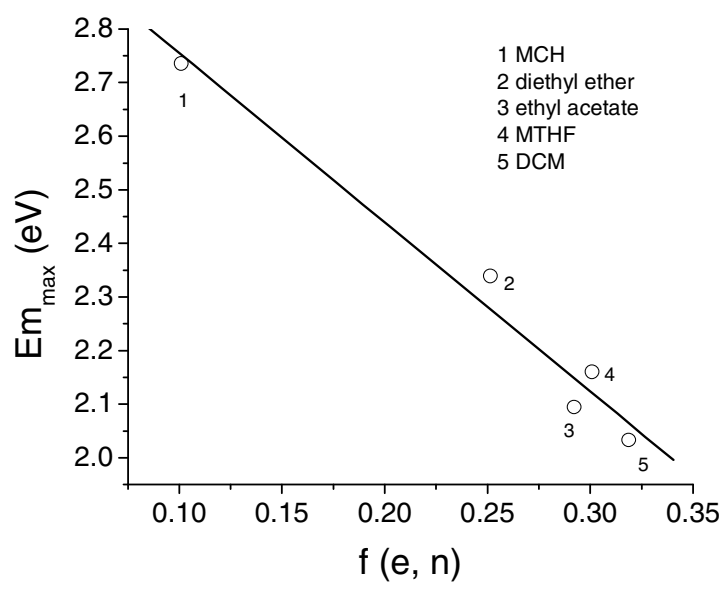

Figure S2. Lippert-Mataga for DMJ-An-tol $\left(\mathrm{R}^{2}=0.985\right.$ where $\mathrm{f}(\varepsilon, \mathrm{n})=[(\varepsilon-1) /(2 \varepsilon+1)-$ $\left.0.5 \times\left(n^{2}-1\right) /\left(2 n^{2}+1\right)\right]$. The solvents are (1) methylcyclohexane, (2) diethyl ether, (3) 2methyltetrahydrofuran, (4) ethyl acetate, and (5) methylene chloride.

\section{References:}

(1)De Barry Barnett, E.; Cook, J. W.; Matthews, M. A. Recueil des Travaux Chimiques des Pays-Bas et de la Belgique 1925, 44, 217-223.

(2)Mee, S. P. H.; Lee, V.; Baldwin, J. E. Chem.--Eur. J. 2005, 11, 3294-3308. 